\title{
Impact of Operational Research on Performance Control of a Project in the Oil and Gas Industry
}

\author{
Ionuț Nica
}

\begin{abstract}
The explosive development of the human society in contrast to the limited character of resources determines the need for successful implementation of mathematic models in the decision-making process concerning the use of available resources. The oil industry includes a series of global processes such as mining, extraction, refining, transport (road, rail, ship and pipeline) and oil products. The products of this industry with the highest degree of utilization are gasoline and diesel but the portfolio is much broader, kerosene, bitumen, fuel and raw materials for other chemicals such as solvents, pesticides, fertilizers and materials plastic. The oil industry comprises three major areas: "upstream" extraction; refining "midstream" and transportation and marketing of downstream products. In most cases refining is considered to be part of downstream, Oil and petroleum products are essential for many industries and their importance is vital in maintaining and developing the industrial area in the current configuration.
\end{abstract}

Index Terms-MATLAB, Monte Carlo, Operational Research, Project Management.

\section{INTRODUCTION}

Technological processes in the oil industry, if the parameters come out of the operational tire, can produce accidental pollution of the soil, water and the atmosphere.

Both the raw material and the products obtained by their physic-chemical characteristics are substances and mixtures dangerous to the environment, thus representing pollutants in the event of loss of operational control, spillage of petroleum products may occur on land and in water. Moreover, the emissions resulting from the oil processing processes create pollution of the atmosphere.

Certain studies show that these emissions, or $\mathrm{CO} 2$, are a source of climate change and dramatic damage to the ozone layer. In addition, the processes defining this industrial sector result in hazardous waste for which it is necessary to find ways of final disposal.

Final disposal can be achieved by recycling, final disposal in specially designed hazardous waste disposal sites, combustion or bioremediation. In view of the above, the oil market is a vast and interesting area that can serve as a context for the application of a wide range of economic methodologies, as well as other disciplines such as operational research and complex project management.

It is very important that in any project, an optimal balance is identified between the initiation, planning, execution, monitoring and control and closure processes applied to managing a team's efforts towards specific objectives and established success criteria.

Published on March 19, 2020.

I. Nica is with Bucharest University of Economic Studies, Romania
A project refers to a form of temporary organization aimed at creating a product, service or unique result. Also, the beginning and the end are well defined and the actions are meant to achieve a specific purpose and objectives in order to achieve a beneficial change or added value.

The temporary nature of the projects is in contrast to the usual activities in a business - repetitive, permanent or semipermanent work undertaken for the production of goods or services. In practice, the management of these two types of systems differs and implicitly involves the development of distinct strategies.

The main challenge in complex project management is the achievement of project objectives within the limits imposed by the various restrictions.

Major categories of constraints refer to purpose, time, quality, and budget. In a second but more ambitious plan, it can be challenged to optimize the allocation of necessary resources and their integration in order to achieve predefined objectives.

The application of project management techniques to the oil and gas industry brings a number of unique challenges through the fact that projects in this sector tend to become increasingly technologically complex.

Therefore, we will continue to apply project management techniques in the context of a specific project in the oil and gas industry, in particular, a project to eliminate hazardous waste, decontaminate the soil and bring the land to its original form.

\section{LITERATURE REVIEW}

The purpose of the methodology described by Ng KokHaur et al. [1] and Naeini et al. [2] is to develop a probabilistic model that determines the performance of a project as well as a control mode that ensures acceptable performance forecasts (budget overruns and planned levels of risk).

The model is developed based on historical data - from previous projects - on performance in terms of cost and time. For each activity a curve is determined - SS and the curve - SS associated with the project is obtained by summing the individual curves. Uncertainty is reflected in this context through Beta distribution functions, associated with cost per activity. These data will be used in a Monte Carlo application to predict the future performance of the project in terms of total cost. According to AbouRizk et al [3], the planning of a large-scale project requires the estimation of the duration of each activity. In order to quantify the uncertainty or random variation of the duration of any activity, a probability density function will be determined. In the literature, it is proposed that this function 
should fulfill the following properties:

The probability density function - denoted $\mathrm{f}(\mathrm{x})$ - must be continuous over an interval $(\mathrm{A}, \mathrm{B})$ where $\mathrm{A}$ is the minimum duration of activity and $\mathrm{B}$ is the maximum duration;

The function $\mathrm{f}(\mathrm{x})$ must be unimodal; so, $\mathrm{f}(\mathrm{x})<\mathrm{f}(\mathrm{m})$ for $\mathrm{x}$ $\neq \mathrm{m}$, where $\mathrm{m}$ is the modal value;

The $\mathrm{A}$ and $\mathrm{B}$ interval ends must be non-negative, distinct and finite so that the duration of the activity is a random and non-generated random variable.

As I said above, Beta distributions for probability distribution functions will be used in this model. Beta distribution has a long history of use in engineering and project management applications.

The main advantage is that this type of distribution can generate a wide variety of shapes through two parameters. The generalized Beta probability distribution is continuous on a finite interval (A, B) and is characterized by two parameters $\alpha$ and $\beta$. Studies show that it is recommended to use such distributions to model random processes within simulation applications.

The generalized Beta probability density function and the Beta function are obtained by equations (1) and (2) respectively:

$$
\begin{aligned}
& f(x ; \alpha, \beta)=\frac{1}{B(\alpha, \beta)} \frac{(x-A)^{\alpha-1}(B-x)^{\beta-1}}{(B-A)^{\alpha+\beta-1}}, A<x<B \\
& B(\alpha, \beta)=\int_{0}^{1} t^{\alpha-1}(1-t)^{\beta-1} d t
\end{aligned}
$$

The simulation is used to generate Static Curves - S (Curves - SS) and is based on the variability of the duration and cost of individual project activities. As Barraza et al. [4], [5] argues, curves - SS offer probability distributions for the budget and the time needed to complete a project at any intermediate stage.

The method for determining budget and time distributions is developed by Kim and Reinschmidt [6] and consists of three steps: (1) generating 'start' distributions for model parameters, (2) adapting model parameters based on reported data, and (3) using the updated forecasting model.

The Monte Carlo simulation can predict the duration and cost of a project based on the variability in the duration and cost of each activity. After estimating probability distributions for costs and duration of activities, a Monte Carlo application will determine the probability distribution of total cost and total duration.

For each Monte Carlo simulation, a value is randomly chosen for each variable included in the analysis, from a predetermined interval, in correlation with its probability of occurrence. This process is repeated several times, typically 1000 iterations are run, and returns a range of potentially achievable results. The method was applied by Molenaar [7].

\section{CASE StUdy}

The project under consideration is a project in the oil and gas industry, consisting of the elimination of hazardous waste, soil decontamination and bringing the land to its original form.[11]

For simplicity, the description of the project will use the terms:
A. Beneficiary, understanding the organization that is legally obliged to remove hazardous waste, decontaminate the infested soil and bring the land to its original form.

B. Contractor, by understanding the company through which the Beneficiary fulfills its legal obligations. During the years of operation, due to technological processes and wastewater treatment, the Beneficiary generated hazardous waste, ie biological sludge. It was deposited in specially designed pools, known as "biological sludge dumps", located on its own but outside the production units, having the following characteristics:

1. Dump 1 - specially designed pool with concrete walls, without waterproof membrane, with a surface of 2 ha and a capacity of $120,000 \mathrm{mc}$. The base of this waste is not concrete. This is located at a distance of approximately $1 \mathrm{~km}$ from the Beneficiary's production units.

2. Dump 2 - a specially designed waterproof membrane with a surface of 1.5 ha and a capacity of $80,000 \mathrm{mc}$. Besides the waterproof membrane, the heap has concrete walls. It is located about 1.5 $\mathrm{km}$ away from the production units of beneficiary.

3. Dump 3 - total concrete basin with an area of 0.75 ha and a capacity of $50.000 \mathrm{mc}$. It is located about $8 \mathrm{~km}$ away from the Beneficiary's production facilities.

Due to the fact that the Beneficiary does not specialize in the disposal of hazardous waste, decontamination of the soil and landing in the initial form, these activities not being in the business profile, in order to achieve the legal requirement, decides to contract these works. The work will be carried out by a specialized international organization with a portfolio of projects. The activities will be carried out only by the Contractor, the Beneficiary having the obligation and the right to monitor the works from the beginning until their completion.

The purpose of the project is to fulfill the legal requirement of the Beneficiary and at the same time to improve the environmental conditions by eliminating the hazardous waste, which is a source of pollution, and bringing the land to the initial form, the objectives of the project are:

A. Final disposal of 250,000 cubic meters of biological sludge;

B. Rehabilitation / decontamination of polluted soil, under biological sludge;

C. Land transfer with an area of 4.25 ha to the initial form, respectively agricultural land, by filling the soil dumps with $30 \mathrm{~cm}$ of it being vegetal soil.

In order to achieve the above purpose and objectives, the Beneficiary has undertaken the following actions:

\section{A. Drawing up the Task Book.}

It included: the purpose, objectives, general data on sludge dumps (location, waste characteristics, land characteristics), summary of the activities necessary to achieve the objectives, general requirements that the organization that will perform the works, respectively the contractor. 
One of the eliminatory requirements was to document the Contractor's experience in at least 10 similar projects, mentioning the time required to complete them, the necessary resources and the results obtained (Referral Letters from the Beneficiaries).

The project specification of the Beneficiary project, i.e. "turnkey project", which means that all the obligations for waste disposal, soil decontamination and land rehabilitation are the Contractor's, the Beneficiary being entitled to monitor its activity through daily inspections, weekly work meetings and monthly progress reports.

In view of this type of project, it was also requested that the respective sites (dumps) be handed over to the Contractor at the beginning of the works, to be taken over by the

Beneficiary upon completion of the works, upon receipt, based on the supporting documents to demonstrate the achievement of the above-mentioned objectives. Payments were also requested to be made only on the basis of the supporting documents issued by the Contractor and accepted by the Beneficiary.

\section{B. Organization of public auction.}

This stage included the following steps: publication of the official announcement and documentation in SEAP (Electronic Public Procurement System); request clarification by bidders and submission of responses by the Beneficiary; submission of tenders and receipt of tenders; the official opening of tenders and the qualification of tenderers respectively; opening and evaluating technical offers; requesting technical clarifications; technical qualification; opening and evaluating financial offers; the final evaluation based on the evaluation criteria set out in the specifications; declaring the winner of the auction.

\section{Contract signing.}

During this stage, Beneficiary - Contractor meetings were organized in order to establish the contractual details and sign the contract.

\section{Delivery - receiving locations (sites).}

The stage consisted in organizing a meeting between the representatives of the Beneficiary and representatives of the Contractor at the dumps 1, 2 and 3, where the delimitation of the areas, their fencing, the establishment of the access roads were made. A Protocol signed by the representatives of both parties, in which obligations have been established in terms of safety at work, compliance with environmental and security obligations, has also been drafted.

Taking into account the contractual obligations established, the Contractor has performed the following actions:

\section{1) Sampling campaign}

It was carried out by the contractor's accredited laboratory, taking samples of sludge and water from different depths, the number of samples being determined according to the legal requirements and the contractor's needs in determining the capacity and characteristics of the sludge processing facility. In total, 400 samples were taken, at 100 points, from the depths of $50 \mathrm{~cm}, 1 \mathrm{~m}, 1.5 \mathrm{~m}$ and $2 \mathrm{~m}$, as follows: 50 waste dumps 1; 35 heap 2 samples; 15 heap samples 3 . At the same stage the samples were analyzed and the results, together with the conclusions derived from them, were sent to the beneficiary.

\section{2) Obtaining building permits and environment}

The Contractor has acquired 4 ha of land in the riverbed area 1 and 2, has prepared the necessary documentation for the construction and environment permits through the authorized firm and at the same time obtained the necessary approvals and agreements to issue them from the competent authorities.

\section{3) Sludge processing facility and bioremediation platform}

The sludge processing facility was a standard facility used by the modular contractor, consisting of: car scales, truck discharge ramps, sludge storage tanks, sludge centrifugation section, water pumping station, wastewater purification ministry, waste incinerator dangerous with on-line emission monitoring at its basket, concrete platform for soil bioremediation.

\section{4) Mobilization and organization of the dump 1}

During this phase of the project, the Contractor arranged a temporary, waterproof membrane platform, near the heap 1 , mounted 3 pumps and the related pipes to extract the sludge from the dumps 1 and 2 (separated only by - a concrete wall) as well as a sludge loader from pools in trucks. Also, a concrete truck platform was built and utilities connections were made to utilities (power, water, steam).

\section{5) Excavating sewage sludge (dump 1)}

Excavation was carried out with the help of pumps, since the hazardous waste was in a mixed mud-water state. It was pumped from the pool through the pipes temporarily mounted to the tapping charger.

\section{6) Shipment of sewage sludge (dump 1)}

The sludge charger mixes the sludge-water mixture into trucks and transported to the processing facility.

\section{7) Sewage sludge processing (dump 1)}

The trucks were weighed at the entrance to the processing facility, then directed to the unloading ramp. The sludge-water mixture was temporarily stored in the tanks and from here pumped to the centrifugal unit. Following centrifugation, the two phases were separated, following two different routes. The water was sent to the purification ministry, treated and then discharged directly into the clean sewer (collector channel belonging to the Beneficiary).

In the case of evacuation there is continuous monitoring of the interlock parameters in the case of exceeding the established limits and automatic shutdown of the whole process. The sludge was sent to the incinerator where it was burned, the ashes being collected. Following collection and laboratory testing, this ash could be mixed with the contaminated and bioremediated soil or sent to a final landfill site (if the heavy metal content was above acceptable limits).

\section{8) Ground waste excavation (dump 1)}

After total removal of the sludge-water mixture, soil at base 1 was excavated to a depth of $10 \mathrm{~cm}$ (depth that was proven by laboratory testing that the soil was contaminated). After excavation, the soil was loaded into trucks and transported to the bioremediation platform where it was treated. 


\section{9) Waste land bioremediation (dump 1)}

The bioremediation process consisted of laying the soil in rows about $1 \mathrm{~m}$ high, aerobic treatment and periodic aeration, using equipment specific to this process.

\section{0) Waste filling and compacting (dump 1)}

After the total excavation of the contaminated soil, the basin was filled with soil. Most of the soil used to fill was purchased from construction companies that were digging for different beneficiaries but at the same time the bioremediated soil was reused. The filling process was carried out in successive stages according to the methodology agreed with the Beneficiary: filling $30 \mathrm{~cm}$, compacting with specific equipment, filling and compacting again. This succession was followed until the basin was brought to the same level as the land in the environment.

\section{1) (Dump 2) sludge excavation}

For details see Action 9;

\section{2) (Dump 2) Sludge}

For details see Action 10;

\section{3) (Dump 2) Sludge Processing}

For details see Action 11;

\section{4) Excavation earth (dump 2)}

For details see action 12;

15) Waste Earth Bioremediation (dump 2)

See Action 13 for details;

16) Fill and Compaction (dump 2)

See Action 14 for details;

17) Mobilization and organization of dumpster site (dump 3)

For details see Action 8;

18) Dump 3 Sludge Excavation

See Action 9 for details;

\section{9) Dump 3 Sludge}

For details see Action 10;

\section{0) Dump 3 Sludge Processing}

For details see Action 11;

There was no need to excavate the earth in the case of dumps 3 because there was no contaminated soil, proven by sampling and analysis in the accredited laboratory.

\section{1) Dump 3 washer}

Concrete walls of the dump were washed by pressure pumps.

\section{2) Dump 3 filling and compacting}

For details, see Action 14;

\section{3) Reception of works}

This action consisted of organizing a meeting between the representatives of the Contractor and the Beneficiary's representatives who, based on the supporting documents drawn up during the project, as well as on the basis of the visit and the findings made in the field in the areas of the 3 dumps. At the reception, the representatives of the competent authorities were also invited to inform about the completion of the works and the state of the land.

\section{4) Delivery - receiving documents}

At this stage, the Joint Beneficiary / Contractor team made a copy / inventory of all supporting documents in the project (e.g.: analysis bulletins, scales, IT data on processed quantities, progress reports, etc.)

\section{5) Delivery - receiving locations (sites)}

On the basis of all the documents received by the Beneficiary as well as of the minutes of the reception of the works, the minutes of the submission of the locations by the Contractor to the Beneficiary were drawn up in accordance with the contractual provisions.

The table below shows the list of activities in the project, as well as the precedent relationships between them, the estimated duration in months and an interval for the cost per activity expressed in euro. Most addictions are classic, finish-to-start (an activity can only begin after the previous activity is over). As far as activities A8, A15, A16, A17 and A21 are concerned, dependencies are also finite-to-start, but there is a $\mathrm{x}$-month lag. As this is negative, the activities in question can start with $\mathrm{x}$ months before the end of previous activities. Another type of addiction present in the studied project is start-to-start (an activity can begin after the previous activity has begun). This is the case for A11, which can begin after approximately one week ( 0.25 months) from the start of previous A10 activity.

TABLE I: MAP OF ACTIVITIES

\begin{tabular}{|c|c|c|c|c|c|}
\hline \multirow{2}{*}{ Activities } & \multirow{2}{*}{ Predecessor } & \multirow{2}{*}{ Description } & \multirow{2}{*}{ Duration (month) } & \multicolumn{2}{|c|}{ Cost (€) } \\
\hline & & & & $\min$ & $\max$ \\
\hline $\mathbf{A 1}$ & - & Draw up specifications & 1.00 & - & - \\
\hline $\mathbf{A 2}$ & A1 & Auction & 4.00 & - & - \\
\hline $\mathbf{A 3}$ & $\mathrm{A} 2$ & Contract signing & 0.25 & - & - \\
\hline $\mathbf{A 4}$ & A3 & Delivering - site reception & 0.03 & - & - \\
\hline A5 & A4 & Campaign sampling and transmission results & 0.50 & 10,000 & 11,000 \\
\hline A6 & A5 & Obtain building permits and environment & 4.00 & 8,000 & 8,500 \\
\hline A7 & A6 & Mud processing facility and bioremediation platform & 7.00 & $3,000,000$ & $3,100,000$ \\
\hline A8 & A7 FS -1 month & Mobilization and organization of the dump 1 & 0.50 & 20,000 & 21,000 \\
\hline A9 & $\mathrm{A} 8$ & Excavating sewage sludge (dump 1) & 8.00 & $1,200,000$ & $1,250,000$ \\
\hline A10 & $\mathrm{A} 8$ & Shipment of sewage sludge - facilities(dump 1) & 8.00 & 600,000 & 620,000 \\
\hline A11 & A10 SS +0.25 months & Processing sewage sludge (dump 1) & 8.00 & $4,200,000$ & $4,250,000$ \\
\hline $\mathbf{A 1 2}$ & $\mathrm{A} 9, \mathrm{~A} 10$ & Excavating earth dumps 1 & 0.50 & 50,000 & 51,000 \\
\hline A13 & A12 & Bioremediation of dirt soil (dump 1) & 3.00 & 150,000 & 151,000 \\
\hline A14 & A13 & Fill filling and compacting ( dump 1) & 0.75 & 270,000 & 280,000 \\
\hline A15 & A11 FS -0.25 months & Excavating sewage sludge dump 2 & 6.00 & 800,000 & 820,000 \\
\hline A16 & A11 FS -0.25 months & Shipment of sewage sludge - facilities (dump 2) & 6.00 & 400,000 & 410,000 \\
\hline A17 & A11 FS -0.25 months & Processing sewage sludge (dump 2) & 6.00 & $2,800,000$ & $2,830,000$ \\
\hline A18 & $\mathrm{A} 15, \mathrm{~A} 16$ & Excavating earth dumps 2 & 0.25 & 35,000 & 36,000 \\
\hline
\end{tabular}




\begin{tabular}{|c|c|c|c|c|c|}
\hline A19 & A18 & Bioremediation of dirt soil (dump 2) & 3.00 & 105,000 & 106,000 \\
\hline $\mathbf{A 2 0}$ & A19 & Fill filling and compacting ( dump 2) & 0.50 & 184,000 & 185,000 \\
\hline $\mathbf{A 2 1}$ & A17 FS -0.5 months & Mobilization and organization of dump 3 site & 0.50 & 20,000 & 21,000 \\
\hline $\mathbf{A 2 2}$ & $\mathrm{A} 21$ & Excavating sewage sludge (dump 3) & 3.00 & 500,000 & 510,000 \\
\hline $\mathbf{A 2 3}$ & A21 & Shipment of sewage sludge - facilities(dump 3) & 3.00 & 500,000 & 510,000 \\
\hline A24 & $\mathrm{A} 21$ & Processing sewage sludge (dump 3) & 3.00 & $1,000,000$ & $1,020,000$ \\
\hline $\mathbf{A 2 5}$ & $\mathrm{A} 22, \mathrm{~A} 23$ & Washing dump 3 & 0.25 & 10,000 & 11,000 \\
\hline A26 & $\mathrm{A} 25$ & Fill filling and compacting ( dump 3) & 0.50 & 140,000 & 142,000 \\
\hline $\mathbf{A 2 7}$ & $\mathrm{A} 14, \mathrm{~A} 20, \mathrm{~A} 24, \mathrm{~A} 26$ & Reception works & 0.03 & - & - \\
\hline A28 & $\mathrm{A} 27$ & Delivery - reciving documents & 0.03 & - & - \\
\hline A29 & $\mathrm{A} 28$ & Delivery - recieving site & 0.03 & - & - \\
\hline
\end{tabular}

The graph of activities can be seen in the figure below. The following representation captures the dependencies between activities, their duration and a series of indicators used to determine the total duration of the project.

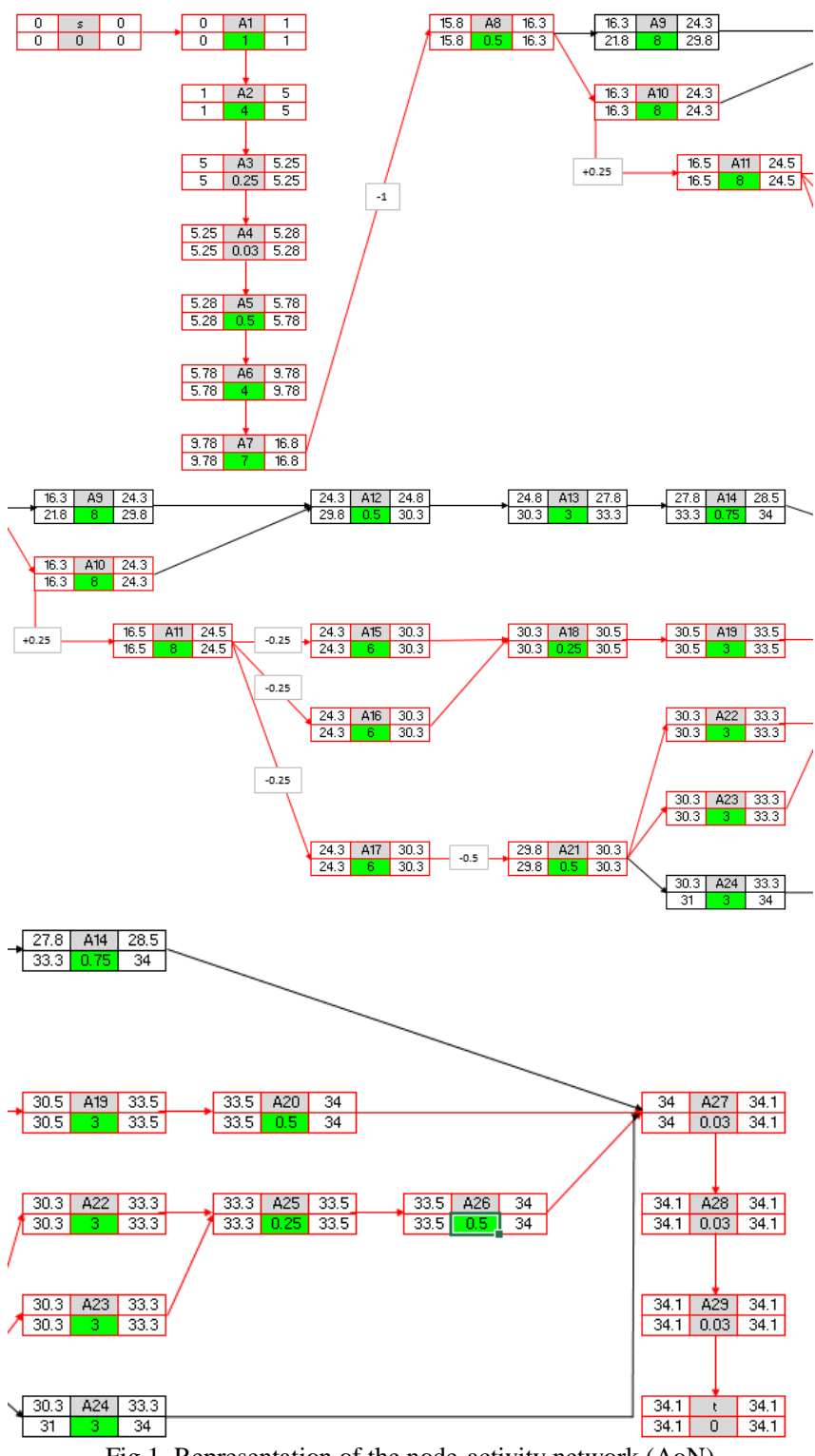

Fig.1. Representation of the node-activity network (AoN)

Based on the above graph, the total duration of the project was 34.12 months. In this respect, the following indicators were also determined for each activity:

- Earliest Start Time (EST) - the earliest date on which to start work

- Earliest Finish Time (EFT) - the earliest date at which the activity can be completed

- $\quad$ Latest Finish Time (LFT) - the latest time to

- complete the activity
- $\quad$ Latest Start Time (LST) - the latest time to start work

We define a critical path as the sequence of activities that describes the longest path within the network. In this case, there are four critical roads, highlighted in Figure 1 by the red color:

1. A1-A2-A3-A4-A5-A6-A7-A8-A10-A11-A15-A18A19-A20-A27-A28-A29;

2. $\mathrm{A} 1-\mathrm{A} 2-\mathrm{A} 3-\mathrm{A} 4-\mathrm{A} 5-\mathrm{A} 6-\mathrm{A} 7-\mathrm{A} 8-\mathrm{A} 10-\mathrm{A} 11-\mathrm{A} 16-\mathrm{A} 18-$ A19-A20-A27-A28-A29;

3. A1-A2-A3-A4-A5-A6-A7-A8-A10-A11-A17-A21A22-A25-A26-A27-A28-A29;

4. A1-A2-A3-A4-A5-A6-A7-A8-A10-A11-A17-A21A23-A25-A26-A27-A28-A29;

Any delay in one of the critical activities determined above will lead to the completion of the project completion deadline.[8][9][10]

One of the requirements that the Beneficiary has imposed in the specifications was the documentation by the Contractor of at least 10 similar projects. On the basis of the information provided, ten series of historical data on the durations of ten types of activities that could be used, according to the methodology described in the previous chapter, resulted in probability distributions that embody the uncertainty associated with each activity within the project. Beta distributions were determined in MATLAB. Thus, in the case of coded activity A5 - Sampling and Transmission Campaign - it has been estimated that the data series can be described by a Beta generalized distribution of parameters $\alpha$ $=1.37$ and $\beta=1.51$, the probability desision function being played by the equation below:

$$
\begin{aligned}
& f(x ; 1.37,1.51)=\frac{1}{B(1.37,1.51)} \frac{(x-5 / 30)^{0.37}(1-x)^{0.51}}{(0.83)^{1.88}}, 5 / 30<x<1 \\
& B(1.37,1.55)=\int_{0}^{1} t^{0.37}(1-t)^{0.55} d t
\end{aligned}
$$

Also, the graphic representation of the above can be seen in the figure below:

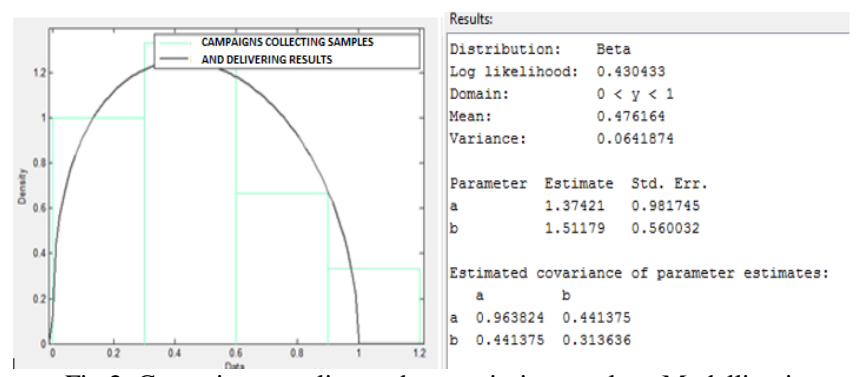

Fig.2. Campaign sampling and transmission results - Modelling in MATLAB

In the case of coded activity A6 - Obtaining Construction 
Authorizations and Environment - it was estimated that the data series can be described by a Beta generalized distribution of parameters $\alpha=1.25$ and $\beta=1.74$, the probability desirability function being given by the equation down:

$$
\begin{aligned}
& f(x ; 1.25,1.74)=\frac{1}{B(1.25,1.74)} \frac{(x-3)^{0.25}(12-x)^{0.74}}{9^{1.99}}, 3<x<12 \\
& B(1.25,1.74)=\int_{0}^{1} t^{0.25}(1-t)^{0.74} d t
\end{aligned}
$$

Also, the graphic representation of the above can be seen in the figure below:

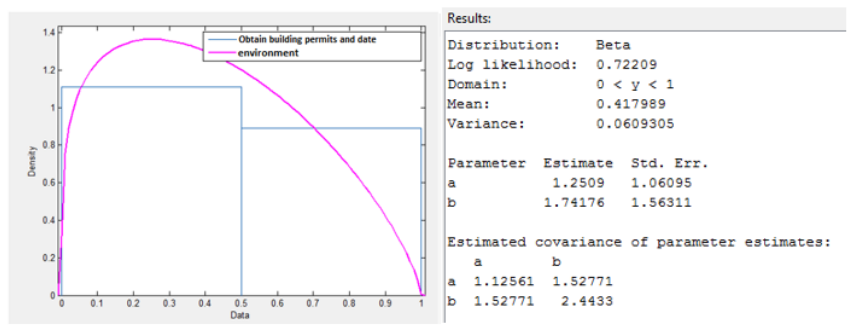

Fig.3. Obtain building permits and environment - Modelling in MATLAB

According to the methodology described above, the probability distributions determined above will be used to construct the assumptions on which the costs per activity may vary throughout the life cycle of the project. As explained in the previous chapters, Beta distributions have a long history of use in project management, and therefore, through the Crystal Ball software, each hypothesis will be based on such a probability distribution.

In this regard, it was necessary to have an interval in which the cost per activity can vary - defined by a minimum and a maximum value - as well as the parameters $\alpha$ and $\beta$ specific to the Beta family distributions. The latter were determined for each type of activity as can be seen above.

Following the Monte Carlo simulation, the results in the figure below were obtained, with the indication that the start-up values for costs per activity were determined to be the averages of Beta generalized distributions. The experiment was based on 1,000 iterations and we can extract the following information:

- The total cost of the project varies between $16,071,572.05$ euros and 16,244,159.77 euros;

- The expected cost of the total cost is EUR $16,142,943.67$, information on the average of the distribution cost the project costs. In other words, this value is based on simulated SS curves and cumulative distribution functions associated with activities. Within each iteration, a value is generated for each cost per activity according to the defined assumptions, the sum of which denotes the value of the simulated total cost. The 1,000 series thus determined reflects the distribution followed by the cost of the project;

- The projected total cost of the project shows a standard deviation of 27,329.69 euros. Another observation regarding the distribution of the total cost of the project is that it tends towards the normal distribution, which is expected from the perspective of the central limit theorem set out in the first chapter. Asymmetry and flattening indicators also confirm this statement - skewness tends to 0 but its positive value indicates a slight asymmetry to the right, while kurtosis tends to 3, its value of 2.92 being specific to a slightly platicist distribution.

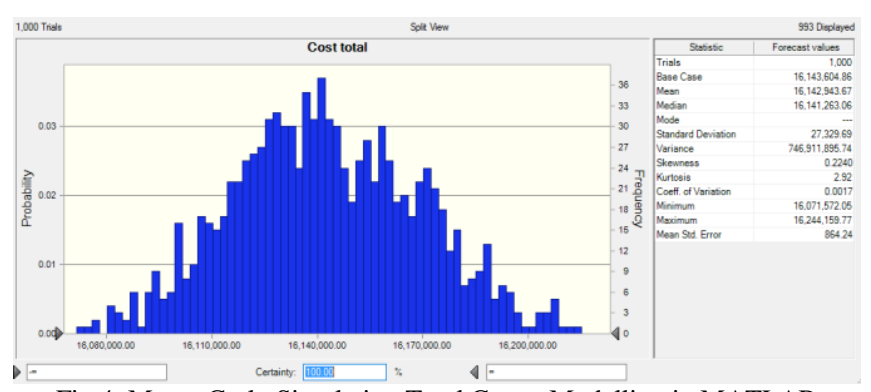

Fig.4. Monte Carlo Simulation Total Cost - Modelling in MATLAB

I conclude that 3 activities account for about $85 \%$ of the total cost variation. Thus, the construction of the sludge processing facility and the bioremediation platform influences $47.6 \%$ of the total cost, the processing of the sludge from the heap 1 has a $24 \%$ impact, while the excavation of the sludge from sludge 1 accounts for $13 \%$.

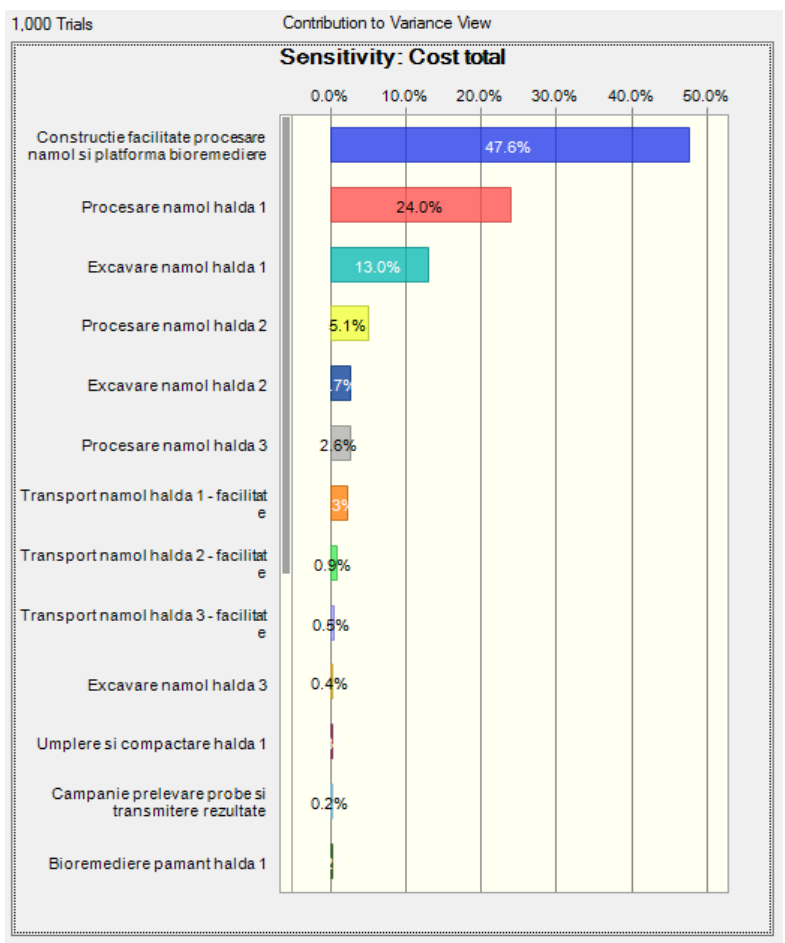

Fig.5. Sensitivity Analysis - Costs. Modelling in MATLAB

Given that the processing facility is a standard, modular facility that requires transport from a European country to Romania, the cost variation is highest due to the different financial regulations of the transition countries, as well as the need for additional elements due to the restrictions (specific emissions sensors, specific interlocks in order to stop the technological process in the case of exceeding the admissible limits).

Considering that when processing the biological sludge from the waste water heap 1 the plant is in technological samples, there may be cost deviations due to higher energy consumption and other utilities (water, technological air).

Due to the fact that the dump 1 is not provided with a waterproof membrane and the base is not concrete, and that it is the oldest of the three, and there is no clear evidence of 
the waste deposited in it, it may be necessary to excavate a larger quantity of sludge.

This argument is also valid for processing, in addition to the argument mentioned in the previous paragraph.

\section{CONCLUSION}

Forecasting is an essential element in project management over the entire life cycle of a project.[12] A complete estimation of the cybernetic effects and control systems engineering on the economy would still be premature at this time.[13]

With the start of a project, the ability to predict its evolution accurately is critical because, even with detailed planning, there are inherent risks that can influence the project's performance. As a result, the project manager is constantly trying to identify some indicators to highlight potential issues so that they can take appropriate action early.

Developing a probabilistic model to determine the performance of a project and a way of control that ensures acceptable performance forecasts can be an effective solution, especially when real performance data is limited. Moreover, simulation applications yield a lot of possible outcomes along with their likelihood of occurrence, thus encompassing the uncertainty element.[14]

Also, this type of solution comes with a number of advantages compared to deterministic methods, exceeding certain limitations. For example, a deterministic analysis treats a limited number of cases, making it difficult to identify factors that significantly affect the end result.

Another impediment encountered in deterministic models is the difficulty of modeling different combinations of input data sets to analyze the effects of a range of possible scenarios.

Thus, in this article, a probabilistic model has been developed to allow for a better control of a project in the oil and gas industry, consisting of the elimination of hazardous waste, decontamination of the soil and bringing the land to its original form.

The model is developed based on historical data - from similar previous projects - on performance in terms of cost and time. Each activity cost is associated with a probability distribution function while the deterministic time remains and is defined as the most probable values.

Uncertainty is reflected in this context through the Beta distribution intensive functions used in project management applications. This is because they can generate a wide variety of shapes through two parameters and provide good results in modeling the behavior of random variables of finite ranges.

This information was concentrated in a Monte Carlo application to predict the future performance of the project in terms of total cost. The resulting sensitivity analysis indicates that approximately $85 \%$ of the total cost variation can be explained by the cost variation of three activities, namely the construction of the sludge processing facility and the bioremediation platform, the sludge processing 1 , followed by the sludge excavation heap 1 .

I conducted a second simulation on predicting the total duration of the project. The Beta distribution functions mentioned above were attached in this case to the duration of the activities. This exercise also allows contrasting the deterministic solution for the total duration and the probabilistic result returned by the Monte Carlo simulation. Thus, an increase of about $22 \%$ for the probability solution relative to the deterministic result was demonstrated.

Also, the main activities that influence the overall duration of the project are the sludge processing in the waste heap 1, obtaining building permits and the environment, followed by the sludge processing of the sludge 2 , their cumulative effect being $82 \%$.

\section{REFERENCES}

[1] Ng Kok-Haur, Koh You-Beng, Pooi Ah-Hin, „Modelling and forecasting count data with a model based on multivariate powernormal distribution: a comparative study with an application", Journal of Economic Computation and Economic Cybernetics studies and research, 2019;

[2] Naeini M. E., Heravi G., "Probabilistic Model Development for Project Performance Forecasting", International Journal of Civil, Environmental, Structural, Construction and Architectural Engineering vol.5, no. 10, p. 428-433, 2011;

[3] AbouRizk S. M., Halpin D. W., Wilson J. R., "Visual Interactive Fitting of Beta Distributions", Journal of Construction Engineering and Management, vol. 117, no. 4, p. 589-605, 1991;

[4] Barraza G. A., Back E., Mata F., "Probabilistic Monitoring of Project Performance Using SS-Curves", Journal of Construction Engineering and Management, vol. 126, no. 2, p. 142-148, 2000;

[5] Barraza G. A., Back E., Mata F., "Probabilistic Forecasting of Project Performance Using Stochastic S Curves", Journal of Construction Engineering and Management, vol. 130, no. 1, p. 25-32, 2004;

[6] Kim B. C., Reinschmidt K. F., 2009, "Probabilistic Forecasting of Project Duration Using Bayesian Inference and the Beta Distribution", Journal of Construction Engineering and Management, vol. 135 , no. 3 , p. 178-186;

[7] Molenaar K. R., 2005, "Programmatic Cost Risk Analysis for Highway Megaprojects", Journal of Construction Engineering and Management, vol. 131, no. 3, p. 343-353;

[8] Crandall K. C., Woolery J. C., 1982, "Schedule development under stochastic scheduling", Journal of the Construction Division, vol. 108, no. 2, p. 321-329;

[9] Gardoni P., Reinschmidt K. F., Kumar R., 2007, “A probabilistic framework for Bayesian adaptive forecasting of project progress", Computer-Aided Civil and Infrastructure Engineering, vol. 22, no. 3, p. 182-196;

[10] Pultar M., 1990, "Progress-based construction scheduling", Journal of Construction Engineering and Management, vol. 116, no. 4, p. 670688

[11] Ward S. A., Lithfield T., 1980, "Cost control in design and construction", New York: McGraw-Hill, ch. 5;

[12] Chirita, N., Nica, I, Cibernetica Firmei. Aplicatii si studii de caz. Economica, 2019;

[13] Nica, I., Chirita, N, Ciobanu, F., "Analysis of Financial Contagion in Banking Networks"," International Business Information Management Association", 2018;

[14] Nica, I., N., Chiriță N., (2020). Conceptual dimensions regarding the financial contagion and the correlation with the stock market in Romania. Theoretical and Applied Economics [Online]. Vol 0(1(622), S), pages 75-86, Spring. Available:http://www.ectap.ro/conceptual-dimensions-regarding-thefinancial-contagionand-the-correlation-with-the-stock-market-inromania-ionut-nica_nora-chirita/a1439/

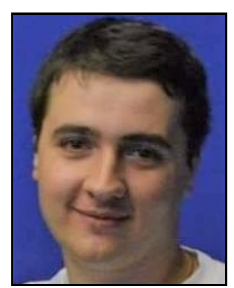

Ionut NICA (b. May 2, 1992) has graduated from the Faculty of Cybernetics, Statistics and Economic Informatics at the Bucharest University of Economic Studies in 2014. He followed a master's degree in Cybernetics and Quantitative Economics, within the same faculty. Currently, he is a $\mathrm{PhD}$ student and teaching assistant in the department of the Faculty of Cybernetics, Statistics and Economic Informatics and work in bank as Basel II Expert in the department of Retail Credit Risk Methodology and Validation. He has high interest in areas such as Cybernetics, Operational Research, Economic Dynamics, Applied Mathematics and Big Data 